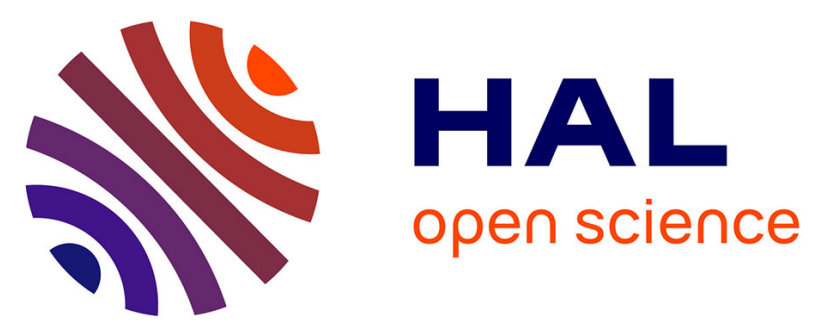

\title{
Towards a Reference Architecture for Swarm Intelligence-based Internet of Things
}

\author{
Ouarda Zedadra, Claudio Savaglio, Nicolas Jouandeau, Antonio Guerrieri, \\ Hamid Seridi, Giancarlo Fortino
}

\section{- To cite this version:}

Ouarda Zedadra, Claudio Savaglio, Nicolas Jouandeau, Antonio Guerrieri, Hamid Seridi, et al.. Towards a Reference Architecture for Swarm Intelligence-based Internet of Things. International Conference on Internet and Distributed Computing Systems, Dec 2017, Mana Island, Fiji. hal-02317288

\section{HAL Id: hal-02317288 \\ https://hal.science/hal-02317288}

Submitted on 15 Oct 2019

HAL is a multi-disciplinary open access archive for the deposit and dissemination of scientific research documents, whether they are published or not. The documents may come from teaching and research institutions in France or abroad, or from public or private research centers.
L'archive ouverte pluridisciplinaire HAL, est destinée au dépôt et à la diffusion de documents scientifiques de niveau recherche, publiés ou non, émanant des établissements d'enseignement et de recherche français ou étrangers, des laboratoires publics ou privés. 


\title{
Towards a Reference Architecture for Swarm Intelligence-based Internet of Things
}

\author{
Ouarda Zedadra $^{1}$, Claudio Savaglio ${ }^{2}$, Nicolas Jouandeau ${ }^{3}$, Antonio Guerrieri ${ }^{4}$, Hamid \\ Seridi $^{1}$, and Giancarlo Fortino ${ }^{2,4}$ \\ 1 LabSTIC, 8 may 1945 University. P.O.Box 401, 24000 Guelma, Algeria \\ \{zedadra_nawel1, seridihamid\} @yahoo.fr \\ 2 DIMES, Università della Calabria. Via P. Bucci, cubo 41c-87036. Rende, Italy \\ csavaglio@dimes.unical.it, giancarlo.fortino@unical.it \\ 3 LIASD, Paris 8 University. Saint Denis 93526, France \\ neai.univ-paris8.fr \\ 4 CNR - National Research Council of Italy Institute for High Performance Computing and \\ Networking (ICAR) Via P. Bucci 7-11C - 87036 Rende(CS) - Italy \\ antonio.guerrierieicar.cnr.it
}

\begin{abstract}
The Internet of Things (IoT) represents the global network which interconnects digital and physical entities. It aims at providing objects with intelligence that allows them to perceive, decide and cooperate with other objects, machines, systems and even humans to enable a whole new class of applications and services. Agent-Based Computing paradigm has been exploited to deal with the IoT system development. Many research works focus on making objects able to think by themselves thus imitating human brain. Swarm Intelligence studies the collective behavior of systems composed of many individuals who interact locally with each other and with their environment using decentralized and selforganized control to achieve complex tasks. Swarm intelligence-based systems provide decentralized, self-organized and robust systems with consideration of coordination frameworks. We explore in this paper the exploitation of swarm intelligence-based features in IoT-based systems. Therefore, we present a reference swarm-based architectural model that enables cooperation among devices in IoT systems.
\end{abstract}

\section{Introduction}

The Internet of Things (IoT) is a highly dynamic and distributed networked system, composed of several cyberphisycal objects producing and consuming information [1]. The IoT enables objects to communicate and to cooperate with each other [2], sensing and analyzing the environment and subsequently performing reasoned actions to achieve their objectives, being therefore defined Smart Objects (SO) [3]. However, such SO-oriented IoT raises many issues involving SO programming, IoT system architecture/ middleware and methods/methodologies for the development of SO-based applications [4]. Agent-Based Computing (ABC) paradigm has been used to deal with such issues [5]. A first use of agents is to exploit smart agents to mitigate the lack of reasoning and intelligence in things in the IoT systems [6]. The basic idea is to embed 
reasoning and intelligence capabilities in each thing or to imitate Swarm Intelligence (SI)-based systems by modeling the system as a collection of simple interacting individuals.

SI envelops a wide variety of algorithms seeking self organization in biological systems inspired by the collective behavior of social insects. It considers simple individuals with limited computational and perception capabilities, indirect and efficient communication tools. In recent years, there has been increased research interest into swarm-based approaches and they have been found to be effective in dealing with several NP-hard problems [7].

The existing IoT architectures do not consider some of the important features in complex systems such as: self-organized and self-adaptive control, the modeling of components as heavyweight agents produce expensive costs and complex implementation, coordination mechanisms used are complex. SOs do not allow cooperation in complex IoT systems. Moreover, the existing SI-based architecture are domain oriented (health, smart cities etc) and there exists a lack on a reference SI-based architecture in literature. To this purpose, a reference model is required to support seamlessly the use of SI features and the integration of SI-based algorithms within IoT systems.

To address the needs above, this paper introduces a new reference swarm-based model for IoT-based systems. We propose to use: (1) Multi-Agents Systems (MAS) technology (stationary and mobile, heavyweight and lightweight agents) to mitigate the lack of reasoning and intelligence in things and to exploit the benefits of edge computing by processing data in local, (2) Cloud Computing environment (CC) for its benefit in storage and computational resources, (3) stigmergic communication through shared workplace (agents) to provide cooperation between SOs without the need to know each other, to overcome the limited memory in things, to lower costs and reduce bandwidth requirements, (4) self-adaptive and self-organized behaviors in different layers in order to meet the fast changes in IoT data flow and device integration. This architecture aims to hide heterogeneity between devices and to cope with complexity and real-time issues. Since this work is a preliminary contribution, adaptive and decentralized algorithms could be included in the MASs of each layer for developing large-scale cyberphysical applications.

This paper is organized as follows. In section 2 related works on SI-based algorithms and agent-based IoT architecture are briefly introduced. In section 3 , details of a SI-based IoT architecture are discussed, in which stigmergic interaction among MASs in different layers is emphasized. Concerns and challenges of IoT are discussed in section 4 followed by a conclusion in Section 5 .

\section{Related Work}

We divide this Section into three Sections. In Section 2.1 we briefly introduce the SI paradigm. In Section 2.2, we give an overview about SI-based algorithms and their use in IoT-based systems. In Section 2.3 we present the most relevant agent-based IoT architectures and framework. 


\subsection{SI Paradigm}

SI is an artificial intelligence technique based around the study of collective behavior in decentralized, self-organized systems [8]. It is defined as any attempt to design algorithms or distributed problem-solving devices inspired by the collective behavior of some social living beings, such as ants, termites, birds, and fishes [9]. The main features of SI-based algorithms that make them appealing for the development of IoT applications include scalability, robustness, parallel action, flexibility [10].

\subsection{SI-based Algorithms}

Several algorithms inspired by the collective behavior of social insects or flocking birds have been proposed in the literature and applied successfully to many real world applications such as the benchmark mathematical problems, optimization tasks, routing network, image processing, data mining, scheduling tasks, and collective robotics.

The Ant Colony (AC) algorithm is another example of SI which is inspired by the classical ant system. The pheromone deposit and pheromone evaporation are used in [11] to calculate the trust value in the process of selecting trustable objects [12] to solve the path routing problem of the urban traffic in an IoT system [13].

The Ant Colony Optimization (ACO) is a population-based meta-heuristic [14] that can be used to find approximate solutions to difficult optimization problems. In [15], MAS architecture and ACO algorithm are used to solve routing problems. Authors in [16] use the classical ACO with some adjustments to pheromone trail to deal with disruption.

Particle Swarm optimization (PSO) is a population-based stochastic optimization technique for the solution of continuous optimization problems. Its mechanism is inspired by the coordinate movement of fish schools and bird flocks [17]. PSO is used in [18] to provide a fast recovery mechanism from path failure due to energy depletion or physical damage with an alternative path. It chooses a path with the optimal fitness from the optimal sensor nodes. In [19], The inertia weight method of PSO is applied in the encoding scheme to evaluate the total revenue to find good solutions where a particle is represented by the raw material inventory level in manufacturer.

Artificial Bee Colony algorithm is an optimization algorithm inspired by the intelligent foraging behavior of honey bee swarm [20]. In [21], Artificial Bee Colony algorithm is used to achieve the optimal solution services in an acceptable time and high accuracy where authors propose a service model and use Artificial Bee Colony algorithm to accomplish its instantiation. In [22], authors consider service optimization problem. They propose a set of service domain-oriented Artificial Bee Colony algorithms based on the optimization mechanism of Artificial Bee Colony and the influence of the service domain features.

Honey Bee Mating Optimization (HBMO) is considered as a typical swarm-based approach to optimization, in which the search algorithm is inspired by the process of marriage in real honey-bee [23]. In [24], it is used in routing problem in cognitive radio sensor networks. 


\subsection{Agent-based IoT Architectures and Framework}

Fortino et al. [25] propose a multi-layered agent-based architecture which allows for developing proactive, context-aware smart objects, atop heterogeneous computing and sensor/actuator platforms and coordinate them through a well-defined Jade-based middleware. In [26], authors propose a cloud-assisted and agent-oriented IoT architecture that will be realized through ACOSO [27] and agent-oriented middleware for cooperating smart objects, and body cloud, a sensor-cloud infrastructure for large scale sensorbased systems. A scalable architecture capable of dealing with all the kinds of sensor in a smart city regardless of the technology used is proposed in [28]. Authors consider lightweight and heavyweight agents for the integration of heterogeneous sensors and for information fusion respectively. Authors in [29] present iSapiens, which is an IoTbased platform for the development of general cyberÛ́physical systems suitable for the design and implementation of smart city services and applications. The iSapiens platform implements the edge computing paradigm through both the exploitation of the agent metaphor and a distributed network of computing nodes directly scattered in the urban environment.

Godfrey et al. [30] propose a mobile agent framework to achieve communication between heterogeneous devices, search for resources and provide services to devices in the network. The mobile agent is either runs on a dedicated node or computer connected to the network, or embedded in the device itself. Each mobile agent migrate from a node to another using a pheromone-based mechanism [31]. Authors in [32] present Rainbow, an architecture that permits an easy development of smart city applications. It combines MAS and fog computing, so as to allow the creation of distributed and swarm intelligence applications which can interact directly and in real time with the physical world. In [33], a three layered architecture based on multi-agent technology framework to achieve a self-organization and self-adaptation for the future intelligent shop-floor is proposed. It aims at quick organization of production, discovering and dealing autonomously with abnormalities. Authors in [34] propose a three layers framework for IoT (FIoT) based on MAS and machine learning techniques. They use three types of agents: god, adaptive and observer. God agent is used to detect new thing, and to associate to each detected thing an adaptive agent. Adaptive agent is embodied with a thing (the things forms the body, while the agent forms the controller), it makes decisions based on Finite State Machine (FSM) or Artificial Neural Networks (ANN) technologies. Observer agent examines the environment to determine if the system meets its global goals, otherwise it defines new behaviors to adaptive agents. Two different applications are used: quantified things and smart traffic control. Authors in [35] propose ANT, a guiding system for tourists based on the foraging mechanism of ants. ANT enables unique features for tourist navigation by using an artificial stigmergy algorithm. It allows tourists to: (1) the serendipitous discovery while providing the information to the rest of participants, and (2) make route decisions using the data provided by the server. It is based on MAS and uses three software agents: Final user ANT's agent, Objects ANT's Agent and ANT server. 


\section{Proposed Reference Architecture for SI-based IoT}

The proposed architecture is based on Multi-Agent Systems (MAS) and swarm intelligence. One basic feature is to introduce self-organized and self-adaptive behaviors in each layer through stigmergic communication. We present in Section 3.1 the building blocks of the proposed architecture. We describe in Section 3.2 the three layers of the architecture. In Section 3.3 we discuss the common features and novelties regarding the existing architectures.

\subsection{Architectural Building Blocks}

We use two types of agents (lightweight and heavyweight) and Smart Objects (SOs). Figure 1 shows the class diagram representing the main components of the proposed model and the relations between them.

Smart Object (SO) is modeled as lightweight agent which can perceive its environment, act on it, and share information with other SOs. They do not know each other, but they cooperate and communicate indirectly through sharing messages in the shared memory in Local Multi-Agent System (LMAS). SOs have inherent hardware and software constraints, e.g., low processing and transmission power, memory, and battery life. Hence, to have a seamless operation in the IoT, the communication process must support the exchange of information between heterogeneous devices and across heterogeneous networks. Each SO need to subscribe with a subject (a set of key words that trigger some of its behaviors) to the closest LMAS. A SO can; (1) perceive or collect data from its environment, (2) act on its environment, (3) pass on collected data to shared memory in LMASs, (4) update data stored in the shared memory, (5) retrieve stored data in shared memory.

Local Multi-Agent System (LMAS) it is a MAS composed of three agents: A shared memory agent, an Observer agent, and an Adaptive agent. The shared memory agent is a storage memory used to store data sent by different devices. It can be updated by devices or adaptive agents. Data streams provided by SOs can be interpreted as task progress, state indicators or triggers to other SOs. The Observer is a lightweight agent which uses a simple FSM to achieve its tasks. It is responsible of observing the changes in the shared memory agent, whenever there is a new message, it notifies the particular SO if they are directly connected to it, else it adapts its behavior to a mobile agent, it migrates to the appropriate LMAS with the corresponding data. The Adaptive agent is a heavyweight agent, responsible of transforming the received information to meet sensors requirements, in order to provide the architecture with openness regarding the connection to sensor networks of different nature and to be uniform in the upper layers.

Adaptive Multi-Agent System (AMAS) it is a MAS composed of homogeneous adaptive agents. They are responsible of the processing, filtering and extraction of meaning information from a data flow. They deliver the resulting data to specific gateways. Adaptive agents in MAFs do not need to communicate, but rather they share a common memory agent to retrieve data provided by LMASs from the lower-layer. Adaptive agents need to be augmented with learning capabilities, logical reasoning, 
SI-based algorithms or use domain ontology to self-adapt and produce intelligent behaviors. MAF produce self-organize and self-adaptive behaviors. In a MAF, selforganizing behaviors can be produced by shared memory agent which controls the flow of data, each time it changes the organization of MSMA to meet the requirements. Also self-adaptive behaviors are produced by AMAS, which requests new learning, ontology, algorithms whenever a new unknown data is retrieved.

Local Agent Framework (LAF) is a set of LMASs connected through an internet connection. It is located in the local environment besides the devices.

Middleware Agent Framework (MAF) is a set of AMASs which share a common memory agent. It is located in the middleware layer and deployed in the CC.

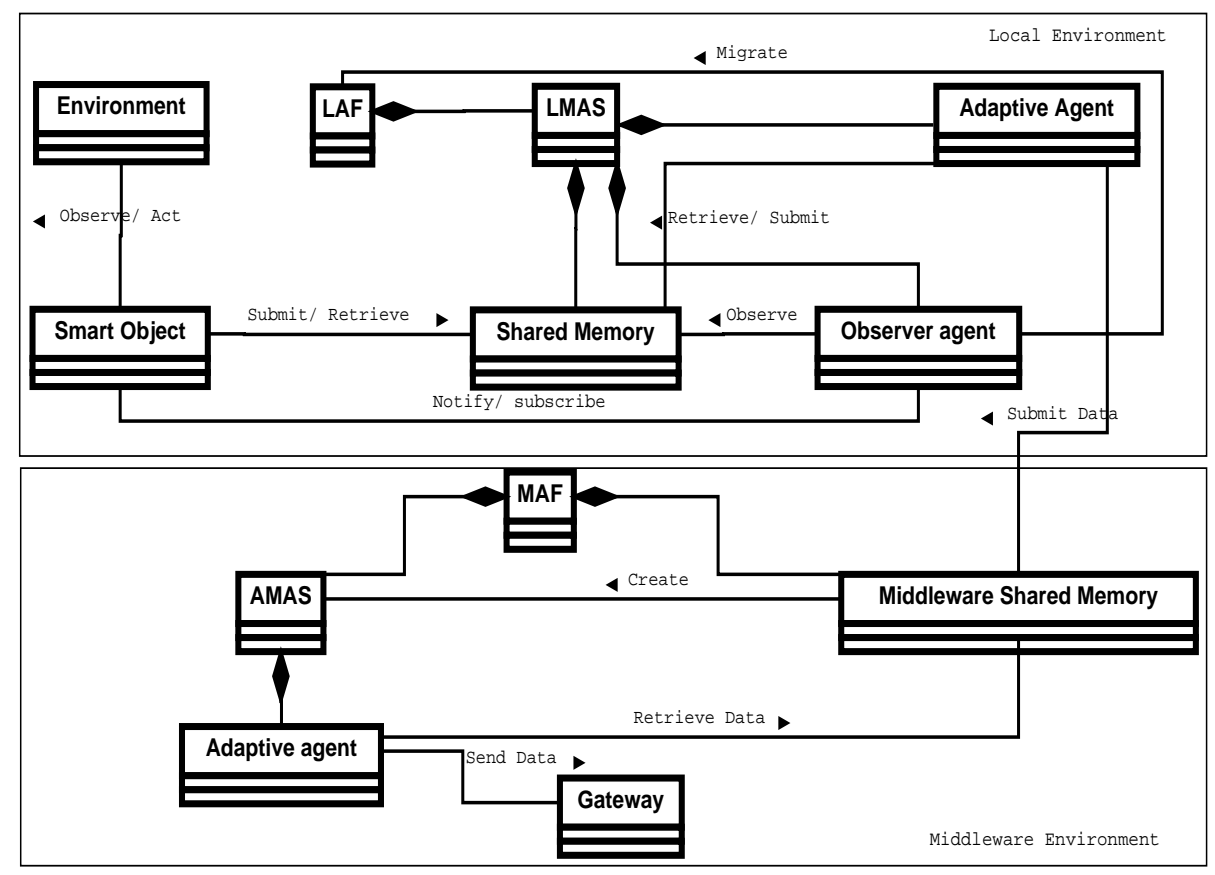

Fig. 1: Class diagram of the proposed SI-based Model

\subsection{Architectural Layers}

The architecture is divided into three layers as shown in Figure 2 , physical layer, middleware layer and application layer. We describe these layers below:

- Physical layer: consists in everyday objects and devices (represented by a SO) connected to LAF agents through diverse communication technologies (RFID, WI-FI, ZigBee...). LAF agents are connected among them via a local network. SO sense 
changes in the environment and transmit a raw of data to LAF agents in order to share them with other SOs to allow a cooperation between them. Recorded data is processed in local via adaptive agents in order to transmit it to other SOs and to upper layers. Using agent technology provides this layer with: (1) an open environment that allows the dynamic addition of new sensor systems and technologies, thus allowing a simple device-to-device communication, (2) a tight conjoining of the top-level intelligent models and the bottom-level physical resources, (3) a local process of data (edge computing), thus reducing costs and bandwidth.

- Middleware layer: this layer gathers data from LAF agents in the lower layer. It consists in a set of MAF agents which use a shared memory to retrieve data submitted by LAF agents. A strategy to create dynamically other MAFs is used to self-organizing to data changes. MAF agents benefit from SI-based algorithms, machine learning, logical reasoning and ontology to transform the collected data into the expected intelligence. This layer is also responsible of the right forwarding of data to its particular gateways to meet their authorized application entities.

- Application layer: includes a variety of potential applications and services enabled by the SI-based IoT systems. Low-level MASs encapsulate data in the form of web services sensitive to context.

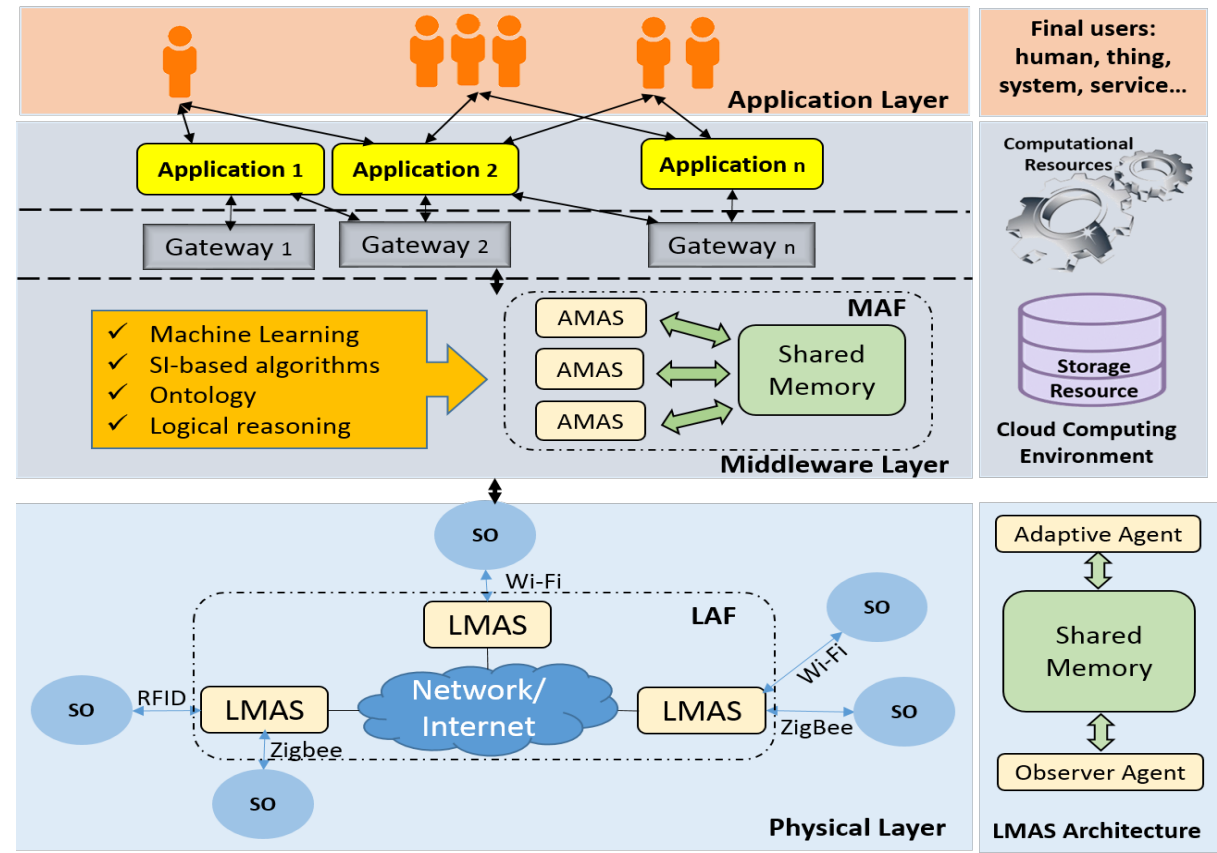

Fig. 2: General design for the SI-based IoT system 


\subsection{Discussion}

As pointed out earlier, the focus of this research is towards evolving swarm intelligence control mechanisms in IoT systems. Hence, the focus is towards developing selforganized and self-adaptive behaviors to control the IoT system. Some of the challenging points addressed in this work and common to existing architectures include:

- Scalability: modeling IoT systems as a swarm provide scalability of the system because the control mechanisms do not depend on the number of devices within the system.

- Service times and bandwidth management: the distributed strategy edge computing allows data processing locally in smart devices rather than being sent to the cloud. It provides action in real time and working within the limits of available bandwidth [32] [36].

- Heterogeneity: in order to solve the problem of heterogeneity among the communicating devices, regardless of the technology used, we use heavyweight agents to release the corresponding transformations [28].

- Intelligence: SI-based algorithms can be used in data processing, such as ACO [14], PSO [17], BFO [37], ABC [20], FFA [38], HBMO [23].

- Big data: CC computational and storage resources will enable storage, access, and processing of the huge amount of data produced by IoT systems [28].

- Devices communication: the proposed architecture allows device-to-device communication through indirect communication between SOs which enables efficient use of network resources [39].

- Coordination: MAS technology provides best solutions to coordinate numerous devices [31] [32].

The proposed architecture considers some features which are not considered in the existing SI-based and non-SI-based ones:

- Coordination: we use stigmergic communication through shared memory to allow cooperation between different components of the IoT system (SOs and agents). The implemented coordination mechanism allows devices and agents to communicate and cooperate without the need of mutual knowledge.

- Self-adaptive behaviors: agents in LMASs are stationary, but can change to be mobile and migrate to other LMASs to transmit data to SOs from other LMASs. Also, the shared memory can manage the number of agents in each AMAS in order to adapt to the dynamic changing of data flow.

\section{Key Challenges and Concerns}

The well known challenges and issues common to most of the research literature on IoT include: standardization, scalability and flexibility, power and energy efficiency, identification, authentication, security and privacy, integration, big data, network communications, inter-operability. Issues related to SI-based IoT systems may include: 
- The adaptation and integration of SI-based algorithms in IoT-based environment to deal with the continue increasing in collected data, to extract meaning data in order to reduce the amount of stored and transmitted data, processing and filtering the huge and heterogeneous data coming from various sensors.

- Network structure of the proposed MAS should allow self-organization of the connected devices [40].

- The co-existence and interaction between physical space and the virtual (data) space in an IoT environment require the development of new technologies to allow data to be processed and manipulated between the real and digital spaces [41].

- The stigmergic communication provides self-organized solutions with lower costs, but its implementation needs somehow the integration of message passing mechanisms. In order to lower costs, resolve congestion and connectivity problems, coordination mechanisms need to avoid the message passing.

- Since IoT connects everyday objects to the Internet, online social networks with personal things information may incur social concerns as well [41].

- In order to meet the IoT system requirements specifically in costs and network efficiency, self-organizing behaviors to manage the number of agents in different layers need to be explored.

- Coordination issues have been widely considered in swarm robotics field and can constitute inspiration to SI-based IoT systems [42].

\section{Conclusion}

The IoT is attracting more and more attentions currently. It involves high-dimensional problems and a large amount of data. SI-based algorithms have proven to be the best algorithms applied to complex, dynamic and large scale systems such those of IoT.

We presented in this paper a reference SI-based model for IoT systems. The model is divided into three layers (physical, middleware and application). We used MAS technology, SI paradigm and edge computing. We modeled the IoT system as a swarm of simple SOs with limited computational and storage capabilities which use stigmegic communication to produce cooperative and complex behavior at the system level. SOs collect and transmit data and act in their environment when necessary. They can cooperate with each other through sharing collected information in a shared memory located in LMASs. Agents are responsible of notifying SOs, transmitting data to other LMASs, adapting and processing data coming from different devices. They use self-organized and self-adaptive behaviors to meet the IoT system requirements.

SI-based algorithms have been considered in numerous research works and they are very promising to integrate intelligence into IoT systems. Modeling IoT systems as a swarm of simple devices with stigmergic communication can provide cheaper and more efficient development solutions. As future work, we intend to implement the proposed reference model and to investigate its application to different IoT systems.

\section{References}

1. D. Miorandi, S. Sicari, F. De Pellegrini, I. Chlamtac, Internet of things: Vision, applications and research challenges, Ad Hoc Networks, 10(7), pp. 1497-1516, 2012. 
2. G. Fortino, A. Guerrieri, M. Lacopo, M. Lucia, W. Russo, An Agent-Based Middleware for Cooperating Smart Objects, International Conference on Practical Applications of Agents and Multi-Agent Systems (PAAMS), pp. 387-398, 2013.

3. G. Fortino, A. Guerrieri, W. Russo, C. Savaglio, Towards a Development Methodology for Smart Object-Oriented IoT Systems: A Metamodel Approach, Intlernational Conference on Systems, Man, and Cybernetics, pp. 1297-1302, 2015.

4. G.Fortino, P. Trunfio, Internet of Things Based on Smart Objects, Technology, Middleware and Applications, ISBN 978-3-319-00490-7, Springer 2014.

5. G. Fortino, Agents meet the IoT: toward ecosystems of networked smart objects, IEEE Systems, Man, and Cybernetics Magazine, 2(2), pp. 43-47, 2016.

6. G. Fortino, W. Russo, C. Savaglio, Agent-oriented Modeling and Simulation of IoT Networks, FedCSIS, pp. 1449-1452, 2016

7. N. R. Sabar, M. Ayob, G. Kendall, R. Qu, A honey-bee mating optimization algorithm for educational timetabling problems, European Journal of Operational Research, 216(3), pp. 533543, 2012.

8. M. Dorigo, M. Birattari, Swarm intelligence, Scholarpedia, 2(9), pp. 1462, 2007.

9. E. Bonabeau, M. Dorigo, G. Theraulaz, Swarm intelligence: from natural to artificial systems, Oxford university press 1, pp. 7, 1999.

10. N. El Zoghby, V. Loscri, E. Natalizio, V. Cherfaoui, Robot cooperation and swarm intelligence, Wireless Sensor and Robot Networks: From Topology Control to Communication Aspects, pp. 168-201, 2014.

11. V. Suryani, S. Sulistyo, W. Widyawan, Trust-Based Privacy for Internet of Things, International Journal of Electrical and Computer Engineering, 6(5), pp. 2396-2402, 2016.

12. Y. Lu, W. Hu, Study on the application of ant colony algorithm in the route of internet of things, International Journal of Smart Home, 7(3), pp. 365-371, 2013.

13. I. Sabbani, M. Youssfi, O. Bouattane, A multi-agent based on ant colony model for urban traffic management, International Conference on Multimedia Computing and Systems (ICMCS), pp. 793-798, 2016.

14. M. Dorigo, G. Di Caro, Ant colony optimization: a new meta-heuristic, Congress on Evolutionary Computation, Vol. 2, pp. 1470-1477, 1999.

15. O. Said, Analysis, design and simulation of Internet of Things routing algorithm based on ant colony optimization, International Journal of Communication Systems, 30(8), 2016.

16. Y. Jiang, Q. Ding, X. Wang, A Recovery Model for Production Scheduling: Combination of Disruption Management and Internet of Things, Scientific Programming, pp. 1-9, 2016.

17. J. Kennedy, R. C. Eberhart, Y. Shi, Swarm intelligence, Kaufmann, San Francisco, 1, pp. 700-720, 2001.

18. S. Luo, L. Cheng, B. Ren, Practical Swarm Optimization based Fault-Tolerance Algorithm for the Internet of Things, KSII Transactions on Internet and Information Systems (TIIS), 8(4), pp. 1178-1191, 2014.

19. C. Fang, X. Liu, P. M.Pardalos, J. Pei, Optimization for a three-stage production system in the Internet of Things: procurement, production and product recovery, and acquisition, The International Journal of Advanced Manufacturing Technology, 83(5-8), pp. 689-710, 2016.

20. D. Karaboga, An idea based on honey bee swarm for numerical optimization, Technical report-tr06, Erciyes university, engineering faculty, computer engineering department, vol. 200, 2005.

21. L. Huo, Z. Wang, Service composition instantiation based on cross-modified artificial Bee Colony algorithm, China Communications, 13(10), pp. 233-244, 2016.

22. X. Xu, Z. Liu, Z. Wang, Q. Z. Sheng, J. Yu, X. Wang, S-ABC: A paradigm of service domainoriented artificial bee colony algorithms for service selection and composition, Future Generation Computer Systems, 68, pp. 304-319, 2017. 
23. B. S. Rani, C. A. Kumar, A Comprehensive Review on Bacteria Foraging Optimization Technique, In Multi-objective Swarm Intelligence, pp. 1-25, 2015.

24. E. Fadel, M. Faheem, V. C. Gungor, L. Nassef, N. Akkari, M. G. A. Malik, S. Almasri, I. F. Akyildiz, Spectrum-aware bio-inspired routing in cognitive radio sensor networks for smart grid applications, Computer Communications, 101, pp. 106-120, 2017.

25. G. Fortino, A. Guerrieri, W. Russo, Agent-oriented smart objects development, International Conference on Computer Supported Cooperative Work in Design (CSCWD), pp. 907-912, 2012.

26. G. Fortino, A. Guerrieri, W. Russo, C. Savaglio, Integration of agent-based and cloud computing for the smart objects-oriented iot, International Conference on Computer Supported Cooperative Work in Design (CSCWD), pp. 493-498, 2014.

27. G. Fortino, A. Guerrieri, W. Russo, C. Savaglio, Middlewares for smart objects and smart environments: overview and comparison, Internet of Things Based on Smart Objects, pp. 127, 2014.

28. P. Chamoso, F. De la Prieta, F. De Paz, J. M. Corchado, Swarm agent-based architecture suitable for internet of things and smartcities, International Conference Distributed Computing and Artificial Intelligence, pp. 21-29, 2015.

29. F. Cicirelli, G. Fortino, A. Guerrieri, G. Spezzano, A. Vinci, An edge-based platform for dynamic Smart City applications, Future Generation Computer Systems (FGCS), 76, pp. 106$118,2017$.

30. W. W. Godfrey, S. S. Jha, S. B. Nair, On a mobile agent framework for an internet of things, International Conference on Communication Systems and Network Technologies (CSNT), pp. 345-350, 2013.

31. W. W. Godfrey, S. B. Nair, A bio-inspired technique for servicing networked robots, International Journal of Rapid Manufacturing, 2(4), pp. 258-279, 2011.

32. A. Giordano, G. Spezzano, A. Vinci, Smart agents and fog computing for smart city applications, International Conference on Smart Cities, pp. 137-146, 2016.

33. Y. Zhang, C.Qian, J. Lv, Y. Liu, Agent and cyber-physical system based self-organizing and self-adaptive intelligent shopfloor, IEEE Transactions on Industrial Informatics, 13(2), pp. 737-747, 2017.

34. N. M. do Nascimento, C. J. P. de Lucena, An agent-based framework for self-adaptive and self-organizing applications based on the internet of things, Information Sciences, 378, pp. 161-176, 2017.

35. López-Matencio P., Vales-Alonso J., Costa-Montenegro E., ANT: Agent stigmergy-based IoT-Network for enhanced Tourist mobility, Mobile Information Systems, Hindawi, 2017.

36. F. Bonomi, R. Milito, J. Zhu, S. Addepalli, Fog computing and its role in the internet of things, 1st Edition of the MCC Workshop on Mobile Cloud Computing, pp. 13-16, 2012.

37. K. M. Passino, Biomimicry of bacterial foraging for distributed optimization and control, IEEE control systems, 22(3), pp. 52-67, 2002.

38. X. S. Yang, X. He, Firefly algorithm: recent advances and applications, International Journal of Swarm Intelligence, 1(1), pp. 36-50, 2013.

39. O. Bello, S. Zeadally, Intelligent device-to-device communication in the internet of things, IEEE Systems Journal, 10(3), pp. 1172-1182, 2016.

40. P. P. Gaikwad, J. P. Gabhane, S. S. Golait, A survey based on Smart Homes system using Internet-of-Things, International Conference on Computation of Power, Energy Information and Communication (ICCPEIC), pp. 330-335, 2015.

41. Y. Qin, Q. Z. Sheng, N. J. Falkner, S. Dustdar, H. Wang, A. V. Vasilakos, When things matter: A survey on data-centric internet of things, Journal of Network and Computer Applications, 64, pp. 137-153, 2016.

42. N. Hoff, R. Wood, R. Nagpal, Distributed colony-level algorithm switching for robot swarm foraging, Distributed Autonomous Robotic Systems, pp. 417-430, 2013. 